\title{
Non-operative management of blunt
}

\section{splenic injuries beyond safety conditions}

\section{A short narrative review}

\author{
Cătălin Aliuș*, Mirela Zivari**, Dan Dumitrescu********, Adrian Cursaru********, Bogdan Șerban*******, \\ Adrian Dumitru*********, Dragoș Șerban**** \\ *General Surgery Department, University Emergency Hospital Bucharest, Bucharest, Romania \\ **Department of Clinical Psychology, University Emergency Hospital Bucharest, Bucharest, Romania \\ ***Orthopaedic Department, University Emergency Hospital Bucharest, Bucharest, Romania \\ $* * * *$ Orthopaedic Department, "Carol Davila" University of Medicine and Pharmacy Bucharest, Faculty of Medicine, \\ Bucharest, Romania \\ $* * * * *$ Department of Pathology, University Emergency Hospital Bucharest, Bucharest, Romania \\ Correspondence to: Mirela Zivari, MD, PhD, \\ Department of Clinical Psychology, University Emergency Hospital Bucharest, \\ 169 Splaiul Independentei Str., Code 050098, Bucharest, Romania, \\ Phone: +40723 302 422, E-mail: mirelaziv@yahoo.com
}

\begin{abstract}
Although the introduction of specific guidelines regarding the acute management of trauma patients have resolved many inconsistencies in the acute treatment pathways, grey areas are still preventing consensus and unitary standards of care. The treatment of splenic injuries has seen a notable shift from splenectomy only in the early $20 t h$ century to mainly non-operative contemporary approaches. However, there is no current agreement on the optimum timing of switching from conservative to operative decisions, raising the legitimate question of whether some patients are put at risk by waiting too much or other patients are deprived of a necessary organ by not waiting enough.

This paper focuses on the non-operative paradigms of blunt splenic injuries and their immediate and long-term clinical implications presented as a short narrative review. It presents the historical perspective on the treatment of splenic injuries, the role of surgery and selective angioembolisation and their repercussions on the immunological functions of the spleen.

Keywords: blunt splenic injuries, non-operative, splenectomy, selective embolisation
\end{abstract}

\section{Introduction}

The management of trauma patients has been constantly improving since the introduction and dissemination of the Advanced Trauma Life Support (ATLS) principles in 1978 [1]. Despite a dynamically updated systematic approach towards the trauma patient, there are still unresolved debates regarding the best course of action in some aspects of the early (not the immediate) management, including that of blunt splenic 
injuries. Over the past century, paradigms related to the standard of care of these patients shifted from immediate surgery, in most cases, towards observation or interventional radiology procedures as the preferred contemporary approach [2]. Nonoperative management has gained wider acceptance with the introduction of standardised assessment, resuscitation, monitoring and complex imaging systems allowing for organ preservation without an increase in morbidity and mortality. New or modified classifications of trauma mechanisms, severity scales and a judicious application of the clinical governance principles helped in contouring boundaries between the operative and non-operative management in blunt splenic injuries.

The aim of this paper was to review the literature regarding the management of blunt splenic injuries and to provide a view on the optimal moment when to decide to abandon NOM in favour of splenectomy. Furthermore, secondary endpoints such as formation of collections, need for blood transfusion, and the number of hospital days reflect various forms of morbidity associated with NOM and should be weighed against the more radical approach. This article was designed as a short narrative review and gives general information on the topic. Further systematic reviews or meta-analyses could provide a more significant statistical view, but the researcher will struggle with inconsistencies in grading, reporting, formulating a specific clinical question, outcome follow up or scarcity of complex data.

\section{Background and historical perspectives}

The secular anecdotical association of the spleen with dark thoughts and aggressive behaviour biased early researchers and anatomists who believed its only role in the body was related to matters of the soul and mind. The English language even retained the archaic word "splenetic" to define an illtempered and angry person. This misconception was backed up by William Harvey, who called the spleen an "ignoble organ". Considering that the early pertinent works on epidemiology and microbiology were only published in the late 19th century, it is obvious why there was very little hesitation in adopting splenectomy as the treatment of choice for splenic injuries [3]. Nobody knew about the functions of the spleen nor did it matter until King and Shoemaker reported, in 1952, the syndrome of overwhelming postsplenectomy sepsis with a high mortality [4]. This was ten years after Alexander Fleming's discovery of penicillin. The increased understanding of the functions of the spleen and the immunological impairment associated with its removal prompted surgeons to revise their traditional approach, hence the first report on non-surgical treatment of a splenic lesion communicated by Upadhaya et al. in 1968, in a paediatric patient [5]. Retrospective research brought up the feasibility of splenic salvage postulated by Billroth, who performed an autopsy on a trauma patient who died of a brain injury and had a concomitant splenic lesion that showed no evidence of recent haemorrhage. Following increasing reports of OPSI from different trauma centres, conservative treatment for splenic injuries in adults started to be timidly embraced with good results, leading to the worldwide adoption of this approach for hemodynamically stable patients.

Almost $10 \%$ of all trauma patients suffer blunt abdominal injuries, the most affected organs being the spleen and liver $[6,7]$. The resistance of any physical object to a change in velocity is defined as inertia. The main mechanisms of injury consist of inertial forces that cause either parenchymal damage or pedicle avulsions. Both situations are 
integrated in the splenic injury classification proposed by the World Society of Emergency Surgery (Table 2). This widely accepted grading of splenic injuries lies at the very foundation of various modern guidelines, offering a common language for all clinicians and radiologists. The most often used classification of blunt splenic injuries proposed by the American Association for the Surgery of Trauma (AAST) (Table 1) only considers the anatomical severity of the lesion, but sometimes patients with severe lesions are stable and might benefit from NOM.

Table 1. AAST classification of splenic lesions

\begin{tabular}{|c|c|c|c|}
\hline Grade of Splenic Injury & \multicolumn{3}{|c|}{ Characteristics of splenic injury } \\
\hline GRADE I & \multicolumn{3}{|c|}{$\begin{array}{l}\text { Subcapsular haematoma involving less than } 10 \% \text { of the surface area, capsular tear, or } \\
\text { parenchymal laceration less than } 1 \mathrm{~cm} \text { in depth. }\end{array}$} \\
\hline GRADE II & \multicolumn{3}{|c|}{$\begin{array}{l}\text { Subcapsular haematoma involving between } 10 \% \text { and } 50 \% \text { of the surface area, } \\
\text { parenchymal haematoma less than } 5 \mathrm{~cm} \text { or parenchymal laceration of } 1-3 \mathrm{~cm} \text { in depth. }\end{array}$} \\
\hline GRADE III & \multicolumn{3}{|c|}{$\begin{array}{l}\text { Subcapsular haematoma involving an excess of } 50 \% \text { of the surface area, ruptured } \\
\text { subcapsular or intraparenchymal haematoma of more than } 5 \mathrm{~cm} \text {, or a parenchymal } \\
\text { laceration larger than } 3 \mathrm{~cm} \text { in depth. }\end{array}$} \\
\hline GRADE IV & \multicolumn{3}{|c|}{$\begin{array}{l}\text { Any injury associated with a splenic vascular lesion or active bleeding within the splenic } \\
\text { capsule or a parenchymal laceration involving segmental or hilar vessels that cause } \\
\text { devascularization of more than } 25 \% \text {. }\end{array}$} \\
\hline GRADE V & \multicolumn{3}{|c|}{ Splenic vascular injuries with hemoperitoneum or shattered spleen } \\
\hline \multicolumn{4}{|c|}{ able 2. WSES Classification of splenic injuries } \\
\hline WSES grade & AAST Grading & Haemodynamic status & WSES class \\
\hline I & $|-| \mid$ & Stable & Minor \\
\hline II & III & Stable & Moderate \\
\hline III & III-IV & Stable & Moderate \\
\hline IV & $\mathrm{I}-\mathrm{V}$ & Unstable & SEVERE \\
\hline
\end{tabular}

\section{Materials and methods}

Papers from 2000 to 2020, related to blunt splenic injuries, were selected from PubMed, using specific interrogations such as: a) blunt splenic injury, b) splenic injury conservative, c) spleen trauma operative. A total number of 1430 papers have been identified, but only systematic reviews, meta-analyses or commented guidelines were selected. This reduced the number to a total of 320 articles (Fig. 1). Case reports and letters were excluded from the study. The main outcome of the review was to assess the choice for operative management and secondary outcomes were a) formation of collections, b) timing of vaccination, c) prevalence of OPSI, d) need for surgery, e) hospital stay, $f$ ) need for embolisation, g) need for transfusion f) early mortality. Two reviewers curated the abstract and tagged one third of the papers marginally relevant to the topic, hence these were not included in the review. 


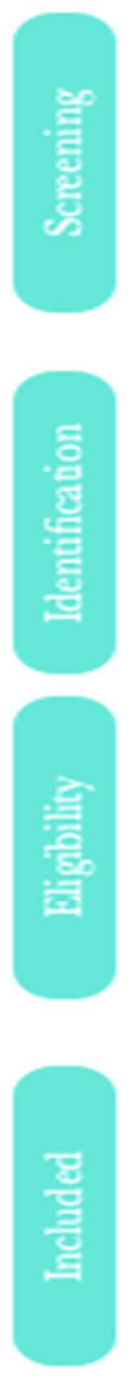

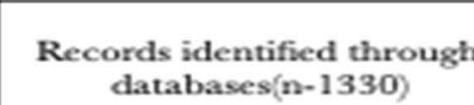
databases $(n-1330)$
Records identified through additional sources $(n-100)$



Fig. 1 PRISMA Diagram

103 papers were considered eligible for the final analysis (Fig. 1). Differences between reviewers were resolved by mutual agreement following discussions. The provenance of the texts and their authors were not blinded to the reviewers.

\section{Results and Discussions}

\section{a) the operative decision}

Non responsive haemodynamic instability was the main contraindication for NOM of splenic injuries and a universally accepted criterion for emergency laparotomy and most often splenectomy [8]. However, the introduction of regional trauma centres led to a reduction in the total number of laparotomies and splenectomies. This situation was best illustrated in the United Kingdom where, according to a recent paper by Yiannoullou, access to specialised care was improved within a regional trauma network, which reduced splenectomy rates with $6 \%$ and increased the use of embolisation therapy from $30 \%$ to $61.2 \%$ [9]. In the US, up to $15 \%$ of the patients with blunt splenic injuries will undergo urgent laparotomies and splenectomies secondary to unresolved haemodynamic instability [10]. The vast majority of grade I and II lesions can be 
managed without surgical intervention even in smaller hospitals, but deferring surgery in higher levels of injury might push the patient outside the safety boundaries [11]. Peitzman demonstrated that mortality in patients with successful NOM was less than $4 \%$ and raised to $16,5 \%$ in those with failed conservative management [12]. This data reflects a possible overstretch of the indications for non-surgical approaches in higher degrees of splenic injuries in organ-salvage centred managements. A recent review published by Coccolini et al. comments on the relative indications of the guidelines and emphasises that sometimes small lesions might be associated with haemodynamic instability and require surgery, while in some cases, high grade injuries may be treated conservatively in stable patients [13]. For a similar reason, Spijkerman and his team devised a protocol for observing patients with splenic injuries. His proposed trial of NOM, which includes a fourhour clinical examination, NPO, no antibiotics, a one-hour BP and pulse oximetry for the first 6 hours, followed by 4 hours of measurements [14]. In addition to that, monitoring BP and pulse is not sufficient to predict haemodynamic instability, hence the use of adjunct different indexes and scales to assess the severity of the injuries. Smith suggested that patients must be observed for 3-5 days before a final decision regarding surgery is made and base excess (BE) is a good predictor for clinical deterioration in trauma patients [15]. The technological advancements in radiology and acute care medicine allowed for an ever-increasing number of patients to benefit from total or partial organ preservation procedures and strategies.

\section{b) selective angiography}

Studying splenic vasculature in more than 850 specimens, Liu suggested that lacerations perpendicular to segmental vessels are less likely to be managed without surgery because they produce tears in the vascular tree, in opposition to those parallel to the vessels [16]. In addition to that, the splenic capsule is thicker in children, explaining why the percentages of organ salvage are higher in this age group and why in similar circumstances adults have a higher risk to undergo emergency laparotomies. In all stable patients, a CT with intravenous contrast is the next logical step in evaluating intra-abdominal injuries. This will establish the grading of the splenic injury and will assess if there is any active bleeding. As a rule of thumb, many authors recommend the use of selective angiography for actively bleeding lesions regardless of their grading, but in most cases active extravasation will correspond to grades IV or $\mathrm{V}$ lesions carrying a higher risk of failure of conservative measures [17]. Observation only in lower grades might predispose to delayed splenic ruptures and increases the percentage of those with failed NOM $[\mathbf{1 8}, \mathbf{1 9}]$. The choice for proximal or distal embolisation is based on the type of injury and has little consequences on postoperative sequelae. Various authors reported conflictual evidence about the reduction in the number of surgeries with the employment of embolisation, but the general trend is to maximise the use of this adjunct to NOM in blunt splenic injuries $[\mathbf{2 0}, \mathbf{2 1}]$. In a large study over a period of 10 years in a trauma centre, D'arcy et al. reported that the need for massive transfusion was around 36\% for those requiring splenectomies, $4.2 \%$ for those observed and nil for patients who benefited from selective angiographic embolisation. This suggests that early radiological treatment contributes to stabilisation and reduces complications associated with transfusion [22].

c) Impact on the immunological function of the body in partial and total splenectomy

Traditionally the asplenic patient is at high risk of developing OPSI in the first years after 
splenectomy due to decreased protection against incapsulated bacteria such as Streptococcus pneumoniae, Haemophilus influenza and Neisseria meningitidis. This is a severe condition with mortality that reached $70 \%$ two decades ago $[\mathbf{2 3 , 2 4}]$. Early start of modern antibiotics and a high index of suspicion lowered the percentage of fatalities in OPSI. Papers related to the lifelong use of low dose penicillins with or without judicious vaccination against the above-mentioned microorganisms are still debated, but many practicians choose a form of postoperative protective measures. Partial splenectomies, splenorrhaphy and selective embolization offered, at least in theory, a way around vaccination or lifelong antibiotic regimens. The function of the residual spleen has been studied using surrogate tests such as levels of immunoglobulins, Howel-Jolly bodies or Technetium labelled erythrocytes. JAG Skimmer et al. analyzed over 800 publications in a recent systematic review and concluded that the splenic function is preserved in various degrees after partial organ sparring procedures, but there is no gold standard test to quantify the function of the spleen [25].

\section{d) existence and reliance on guidelines}

Adherence to guidelines in the management of splenic injuries is higher in paediatric population, hence the lower operative numbers in children presenting with blunt splenic injuries compared to adult patients. If three decades ago in the United States more than half of the adults with splenic injuries were subjected to splenectomies, this number decreased to $17 \%$ in 2010 and was further improved to approximately $12 \%$ due to acceptance and implementation of evidence-based guidelines $[26,27]$. There are no national guidelines in Romania regarding blunt splenic injuries and many centres still do not have local treatment protocols. We believe that questionable clinical decisions, better patient outcomes and judicious use of resources could be achieved by the development of national guidelines.

\section{Conclusions}

Modern selection of patients for NOM of blunt splenic injuries is based on haemodynamic stability rather than a purely anatomical classification, as pointed out by many recent studies. When it comes to a decision for conservative management, rigorous and repeated clinical examinations should be performed following a locally agreed protocol to ensure uniform clinical impressions and help decision making. High turnover centres and hospitals with ICU, transfusion and theatre facilities are reporting higher rates of NOM as opposed to smaller hospitals in which conservative approaches equate sometimes with pushing the patients beyond safety conditions. This calls for a centralisation of trauma hospitals and urgent referral of selected cases. Polytrauma is not a contraindication for non-operative management, but this must be decided strictly in centres with experience in managing trauma patients. There is no unanimously accepted consensus regarding the optimal treatment of blunt splenic injuries, but there is general agreement that clinical common sense and a constant preoccupation for the safety of the patient takes precedent over the temptation for organ preservation approaches.

\section{Conflict of Interest statement}

Authors state no conflict of interest.

\section{Acknowledgements}

None.

\section{Sources of Funding \\ None.}

\section{Disclosures}

None. 


\section{References}

1. Styner R. The Light of the Moon - Life, Death and the Birth of Advanced Trauma Life Support. 2012.

2. Upadhyaya P. Conservative management of splenic trauma: history and current trends. Pediatr Surg Int. 2003; 19:617-627.

3. Sherman R. Perspectives in management of trauma to the spleen: 1979 presidential address, American Association for the Surgery of Trauma. J Trauma. 1980 Jan; 20(1):1-13.

4. King $H$, Shuemaker HB Jr. Splenic studies. I. Susceptibility to infection after splenectomy performed in infancy. Ann Surg. 1952 Aug; 136(2):239-242.

5. Upadhyaya $P$, Simpson JS. Splenic trauma in children. Surg Gynecol Obstet. 1968 Apr; 126(4):781-9o.

6. Fabian TC, Bee TK, Cagianos $C$ et al. Current issues in trauma. Curr Probl Surg. 2015; 39(12):1160-244.

7. Davis JJ, Cohn I, Nance FC. Diagnosis and management of blunt abdominal trauma. Ann Surg. 1976; 183(6):6728.

8. El-Matbouly M, Jabbour G, El-Menyar A, Peralta R, Abdelrahman $\mathrm{H}$, Zarour $A$ et al. Blunt splenic trauma: assessment, management, and outcomes. Surgeon. 2016; 14:52-58.

9. Yiannoullou $P$, Hall $C$ et al. A review of the management of blunt splenic trauma in England and Wales: have regional trauma networks influenced management strategies and outcomes? Ann R Coll Surg Engl. 2017 Jan; 99(1):63-69.

10. Zarzaur BL, Kozar R, et al. The splenic injury outcomes trial: An American Association for the Surgery of Trauma multi-institutional study. J Trauma Acute Care Surg. 2015; 79:335-42.

11. Olthof DC, van der Vlies $\mathrm{CH}$ et al. PYTHIA Collaboration Group. Consensus strategies for the nonoperative management of patients with blunt splenic injury: a Delphi study. J Trauma Acute Care Surg. 2013; 74:156774 .

12. Peitzman $A B$, Heil $B$, Rivera $L$ et al. Blunt splenic injury in adults: Multi-institutional Study of the Eastern Association for the Surgery of Trauma. J Trauma. 2000; 49(2):177-87.

13. Coccolini $F$ et al. Splenic trauma: WSES classification and guidelines for adult and pediatric patients. World J Emerg Surg. 2017 Aug 18; 12:40.

14. Spijkerman R, Teuben MPJ, Hoosain F et al. Nonoperative management for penetrating splenic trauma: how far can we go to save splenic function? World J Emerg Surg. 2017; 12:33.

15. Smith J, Armen S, Cook CH, Martin LC. Blunt splenic injuries: have we watched long enough? J Trauma. 2008 Mar; 64(3):656-63.

16. Liu $D L, X i a ~ S, X u W$ et al. Anatomy of vasculature of 850 spleen specimens and its application in partial splenectomy. Surgery. 1996; 119(1):27-33.

17. Shanmuganathan $\mathrm{K}$ et al. Nonsurgical management of blunt splenic injury: use of CT criteria to select patients for splenic arteriography and potential endovascular therapy. Radiology. 2000; 217(1):75-82.
18. Crichton $\mathrm{JCl}$ et al. The role of splenic angioembolization as an adjunct to nonoperative management of blunt splenic injuries: a systematic review and meta-analysis. J. Trauma Acute Care Surg. 2017; 83(5):934-943.

19. Requarth JA, D'Agostino RB Jr., Miller PR. Nonoperative management of adult blunt splenic injury with and without splenic artery embolotherapy: a meta-analysis. J. Trauma. 2011; 71(4):898-903.

20. Harbrecht BG, Ko SH, Watson GA et al. Angiography for blunt splenic trauma does not improve success rate of non-operative management. J Trauma. 2007; 63:44-49.

21. Wu SC et al. Early selective angioembolization improves success of non-operative management of blunt splenic injury. Am Surg. 2007; 73:897-902.

22. D'Arcy Marsh, Marilyn Day, Amit Gupta et al. Trends in Blunt Splenic Injury Management: The Rise of Splenic Artery Embolization. Journal of Surgical Research. 2021; 265:86-94.

23. Brigden ML. Overwhelming postsplenectomy infection still a problem. West J Med. 1992; 157(4):440-443.

24. Mebius RE, Kraal $G$. Structure and function of the spleen. Nat Rev Immunol. 2005; 5:606-16.

25. Schimmer SJAG, van der Steeg AFW, Zuidema WP. Splenic function after angioembolization for splenic trauma in children and adults: A systematic review. Injury. Int. J. Care Injured. 2016; 47:525-530.

26. Mooney DP, Birkmeyer NJ, Udell JV et al. Variation in the management of paediatric splenic injuries in New Hampshire. Journal of Pediatric Surgery. 1998; 33:107610.

27. Filipescu R, Powers $\mathrm{C}, \mathrm{Yu} \mathrm{H}$ et al. The adherence of adult trauma centers to American Pediatric Surgical Association guidelines on management of blunt splenic injuries. Journal of Pediatric Surgery. 2020; 55(9):1748175328. 